\title{
Value of low-dose dobutamine stress echocardiography on defining true severe low gradient aortic stenosis in patients with preserved left ventricular ejection fraction
}

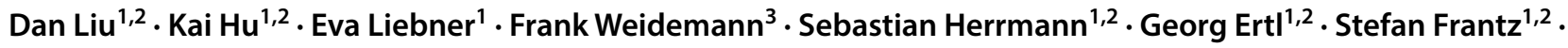 \\ Peter Nordbeck ${ }^{1,2,4}$
}

Received: 18 April 2018 / Accepted: 17 July 2018 / Published online: 23 July 2018

(c) The Author(s) 2018

\begin{abstract}
Low-dose dobutamine stress echocardiography (DSE) is a valuable tool to distinguish true-severe (TS) from pseudo-severe (PS) low gradient aortic valve stenosis (LGAS) in patients with reduced left ventricular ejection fraction (LVEF). However, only scanty studies reported the clinical utility of DSE in differentiating TS-LGAS patients with preserved LVEF. We investigated the clinical utility of DSE in LGAS patients with preserved LVEF and the echocardiographic determinants suggestive of TS-LGAS. 130 consecutive LGAS patients [indexed aortic valve area (AVA) $\leq 0.6 \mathrm{~cm}^{2} / \mathrm{m}^{2}$ and mean trans-aortic pressure gradient $\left(\mathrm{PG}_{\text {mean }}\right)<40 \mathrm{mmHg}$ ] with preserved $(\geq 50 \%, \mathrm{n}=63)$ and reduced $(<50 \%, \mathrm{n}=67) \mathrm{LVEF}$ were included. DSE defined TS-LGAS (projected AVA $\leq 1 \mathrm{~cm}^{2}$ ) in $61.2 \%$ patients with reduced LVEF and in $68.3 \%$ patients with preserved LVEF. Multivariate logistic regression analysis showed that baseline AVA was an independent determinant of TS-LGAS both in $\mathrm{LVEF} \geq 50 \%$ (OR 0.45, $\mathrm{P}=0.004$ ) and $\mathrm{LVEF}<50 \%$ groups ( $\mathrm{OR} 0.55, \mathrm{P}=0.005)$. Reduced septal and lateral mitral annular plane systolic excursion (MAPSE, OR 0.72 and $0.75, \mathrm{P}=0.013$ and 0.016 ) and septal TDI-s' were significantly associated with TS-LGAS in patients with $L V E F \geq 50 \%$. Higher systolic pulmonary artery pressure (SPAP, OR $1.43, \mathrm{P}=0.045$ ) was associated with TS-LGAS in patients with LVEF $<50 \%$. DSE is useful to define TS-LGAS also in patients with preserved LVEF. Lower baseline AVA values are linked with TS-LGAS in both patients with reduced and preserved LVEF. Reduced MAPSE and septal TDI-s' are suggestive of TS-LGAS in patients with preserved LVEF, while higher SPAP is associated with TS-LGAS in patients with reduced LVEF.
\end{abstract}

Keywords Aortic stenosis · Stress echocardiography $\cdot$ Mitral annular plane systolic excursion $\cdot$ Systolic pulmonary artery pressure $\cdot$ Aortic valve velocity ratio

Dan Liu and Kai Hu have contributed equally to this work.

Electronic supplementary material The online version of this article (https://doi.org/10.1007/s10554-018-1416-z) contains supplementary material, which is available to authorized users.

Peter Nordbeck

nordbeck_p@ukw.de

1 Department of Internal Medicine I, University Hospital Würzburg, Würzburg, Germany

2 Comprehensive Heart Failure Center, Würzburg, Germany

3 Medizinische Klinik I des Klinikum Vest, Recklinghausen, Germany

4 Medizinische Klinik und Poliklinik I, Comprehensive Heart Failure Center, University Hospital Würzburg, Oberdürrbacher Str. 6, 97080 Würzburg, Germany

\section{Introduction}

Severe aortic stenosis (AS) is usually defined as an aortic valve area (AVA) $<1.0 \mathrm{~cm}^{2}$, mean transvalvular gradient $\left(\mathrm{PG}_{\text {mean }}\right) \geq 40 \mathrm{mmHg}$, and a peak flow velocity $\geq 4.0 \mathrm{~m} / \mathrm{s}$ [1]. Patients with AVA $<1.0 \mathrm{~cm}^{2}$ and $\mathrm{PG}_{\text {mean }}<40 \mathrm{mmHg}$ are usually defined as low-gradient AS (LGAS). Further examinations are usually required to distinguish true-severe (TS) from pseudo-severe (PS) AS, which is clinically essential for therapeutic decision making because patients with TSLGAS might benefit, whereas PS-LGAS patients might not benefit from aortic valve replacement (AVR) [2]. LGAS is usually found in patients with reduced LV ejection fraction (LVEF $<50 \%$ ), but might also be present in patients with preserved LVEF $\geq 50 \%$ (so called paradoxical LGAS) [3]. Low-dose dobutamine stress echocardiography (DSE) is 
recommended as a tool to define the severity of aortic stenosis in patients with LGAS and reduced LVEF $[4,5]$. To date, data regarding the utility of DSE for defining severity of LGAS in patients with preserved LVEF presenting with either paradoxical low flow or normal flow remain scanty. In the present study, we observed if DSE could also be useful to define AS severity in LGAS patients with preserved LVEF. We further explored the conventional echocardiographic markers suggestive of TS-LGAS in LGAS patients with reduced or preserved LVEF.

\section{Methods}

\section{Study population}

A total of 130 consecutive symptomatic low-gradient AS patients (aged $78 \pm 8$ years, $63.8 \%$ male), referred to the University Hospital Würzburg between January 2011 and December 2016, were included in this study. All patients underwent both standard transthoracic echocardiogram and DSE. Enrollment criteria included indexed aortic valve area (AVAi) $\leq 0.6 \mathrm{~cm}^{2} / \mathrm{m}^{2}$ and mean trans-aortic pressure gradient $\left(\mathrm{PG}_{\text {mean }}\right)<40 \mathrm{mmHg}$ as assessed by transthoracic echocardiogram. The study protocol is shown in Fig. 1. The study was conducted in accordance to the Declaration of Helsinki and was approved by the Local Ethics Committee at the University of Würzburg (AZ 11/03 and 60/14). Informed consent was obtained from all patients or their guardians.

\section{Echocardiography}

Echocardiographic examination was performed with GE Vingmed Vivid 7 or E9, Horten, Norway. Standard measurements on cardiac structural and functional parameters were made according to the current ASE guideline [6]. LV mass indexed to body surface area (LVMi) was calculated by the suggested formula [7]. End-diastolic and end-systolic volumes and LVEF were measured with the biplane Simpson method in the apical 4- and 2-chamber views. Septal and lateral mitral annular plane systolic excursion (MAPSE) and tricuspid annular plane systolic excursion (TAPSE) were measured by M-mode in the apical 4-chamber view. Systolic pulmonary artery pressure (SPAP) was derived from peak tricuspid regurgitation jet velocity using the simplified Bernoulli equation, in combination with the estimated right atrial pressure. LV diastolic function was assessed according to ASE guidelines for the assessment of diastolic function [8]. Tissue Doppler imaging was performed at the septal and lateral mitral annular sites enabling on-line derivation of myocardial systolic velocity (TDI-s') and diastolic early velocity $\left(\mathrm{e}^{\prime}\right)$ [9]. Speckle tracking imaging analysis was performed using EchoPAC software (GE, Horten, Norway) as

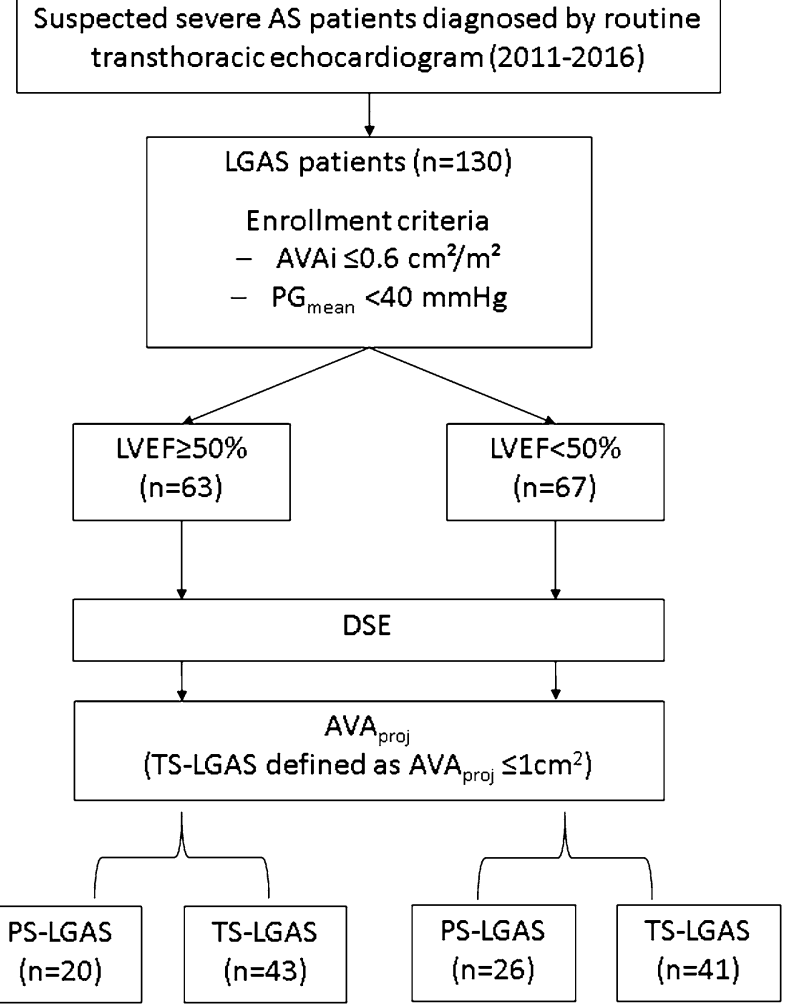

Fig. 1 A flow-chart of the study protocol. LGAS low gradient aortic stenosis, $A V A i$ indexed aortic valve area, $P G_{\text {mean }}$ mean trans-aortic pressure gradient, $L V E F$ left ventricular ejection fraction, $T S$ truesevere, $P S$ pseudo-severe

described previously [10]. Longitudinal peak systolic strain (LS) of each segment was measured and global LS values were acquired by averaging strain rate and strain values of all 18 segments.

\section{Evaluation of AS severity by standard echocardiography}

The diagnosis and classification of AS was made according to recent EAE/ASE recommendations [11]. Left ventricular outflow tract (LVOT) diameter was measured in the parasternal long-axis view focusing on the LVOT at baseline, and this value was also used to calculate the aortic valve area during DSE. The outer edge of the velocity spectrum obtained by continuous wave (CW) Doppler across the aortic valve $(\mathrm{AV})$ was traced to obtain the maximum velocity $\left(\mathrm{V}_{\max }\right)$, maximum and mean trans-aortic pressure gradient $\left(\mathrm{PG}_{\text {mean }}\right.$ and $\left.\mathrm{PG}_{\text {max }}\right)$, and AV velocity time integral (VTI). The subvalvular $\mathrm{V}_{\max }\left(\right.$ LVOT $\mathrm{V}_{\max }$ ), VTI and stroke volume (SV) were obtained by tracing the outer edge of the velocity spectrum at the LVOT by the pulsed wave (PW) Doppler. Aortic valve area by continuity equation VTI [AVA (VTI)] was automatically calculated using the formula: 
$3.14 / 4 *\{\text { LVOT Diam }\}^{2} *\{$ LVOT VTI $\} /\{$ AV VTI $\}$.

Valvuloarterial impedance (Zva) was calculated using the formula:

\section{Results}

\section{Clinical characteristics}

$\mathrm{Zva}\left(\mathrm{mmHg} / \mathrm{ml} / \mathrm{m}^{2}\right)=\left\{\right.$ systolic blood pressure $\left.+\mathrm{PG}_{\text {mean }}\right\} /$ body surface area indexed SV $\}$.

\section{Low-dose dobutamine stress echocardiography}

Dobutamine infusion was started at a dose of $5 \mu \mathrm{g} / \mathrm{kg} / \mathrm{min}$, then increasing to 10,15 , and $20 \mu \mathrm{g} / \mathrm{kg} / \mathrm{min}$ at 3 -min intervals. Blood pressure and heart rate were recorded at rest and at the end of each stage of dobutamine dosage. Contractile reserve was defined as an increase in stroke volume (SV) of $20 \%$ or more [12]. The projected aortic valve area at normal transvalvular flow rate $\left(\mathrm{AVA}_{\text {proj }}\right)$ was calculated using a simplified method with the following formula [13]: Simplified $\mathrm{AVA}_{\text {proj }}=\mathrm{AVA}_{\text {rest }}+\mathrm{VC}_{\text {simpl }} \times\left(250-\mathrm{Q}_{\text {rest }}\right)$, where $\mathrm{VC}_{\text {simpl }}$ is the valve compliance and $\mathrm{Q}$ was computed by dividing $\mathrm{SV}$ by $\mathrm{LV}$ ejection time. $\mathrm{VC}_{\text {simpl }}$ was computed with the formula:

$\left(\mathrm{AVA}_{\text {peak }}-\mathrm{AVA}_{\text {rest }}\right) /\left(\mathrm{Q}_{\text {peak }}-\mathrm{Q}_{\text {rest }}\right)$,

where $\mathrm{AVA}_{\text {rest }}$ and $\mathrm{AVA}_{\text {peak }}$ are AVA at rest and peak DSE. $\mathrm{Q}_{\text {peak }}$ and $\mathrm{Q}_{\text {rest }}$ are $\mathrm{Q}$ at rest and peak DSE. TS-LGAS was defined as $\mathrm{AVA}_{\text {proj }} \leq 1 \mathrm{~cm}^{2}$.

\section{Follow-up}

Patients were followed up for a mean of 18 (12-27) months by clinical visit or telephone interview.

\section{Data analysis}

Continuous variables are presented as mean \pm standard deviation or median (interquartile range, IQR), as appropriate. Normal distribution of all continuous variables was tested by inspecting skewness, kurtosis, and Q-Q plots. Clinical and echocardiographic continuous variables between preserved and reduced LVEF groups and between PS-LGAS and TSLGAS groups were compared using independent Student's $\mathrm{t}$ test or Mann-Whitney U test as indicated. Categorical variables were expressed as percentages and were compared using a Chi square test or Fisher's exact test, as appropriate. Multivariate logistic regression analysis was conducted to identify markers for differentiating TS-LGAS from PSLGAS. Odds ratios (OR) with $95 \%$ confidence interval (CI) were assessed for indicating diagnostic performance. A twotailed probability value $<0.05$ was considered significant. Statistical analysis was performed using IBM SPSS, version 23 for Windows (IBM Corp., New York, USA).
Patients were divided into two subgroups: $\mathrm{LVEF} \geq 50 \%$ group $(n=63)$ and LVEF $<50 \%$ group $(n=67)$. As shown in Table 1, the proportion of male patients was significantly higher in the LVEF $<50 \%$ group than in the LVEF $\geq 50 \%$ group $(82.1 \%$ vs. $44.4 \%, \mathrm{P}<0.001)$. Prevalence of NYHA class III-IV was documented in $79.1 \%$ of LVEF $<50 \%$ patients and $55.5 \%$ of $\mathrm{LVEF} \geq 50 \%$ patients $(\mathrm{P}=0.003)$. The prevalence of diabetes $(52.2 \%$ vs. $33.3 \%, \mathrm{P}=0.030)$ and coronary heart disease $(71.6 \%$ vs. $49.2 \%, \mathrm{P}=0.009)$ was also significantly higher in the LVEF $<50 \%$ group than in the $\mathrm{LVEF} \geq 50 \%$ group.

\section{Baseline echocardiographic characteristics}

Mean LVEF was $60 \pm 6 \%$ in the LVEF $\geq 50 \%$ group and $46 \pm 4 \%$ in the LVEF $<50 \%$ group (Table 2). Patients with LVEF $<50 \%$ had larger LV, RV and LA cavities, thicker LV walls, lower septal and lateral MAPSE and TAPSE as compared to patients with $\mathrm{LVEF} \geq 50 \%$ (all $\mathrm{P}<0.05$ ). AV Vmax, $\mathrm{PG}_{\text {mean }}$, LVOT $\mathrm{V}_{\text {max }}$, AV velocity ratio, and SV were significantly lower, while Zva was significantly higher in the LVEF $<50 \%$ group than in the LVEF $\geq 50 \%$ group (all $\mathrm{P}<0.05$ ). AVA VTI was similar between the LVEF $\mathrm{LVEF} \geq 50 \%$ and $<50 \%$ groups $(0.86 \pm 0.14$ vs. $\left.0.84 \pm 0.16 \mathrm{~cm}^{2}, \mathrm{P}=0.538\right)$. LV longitudinal systolic function parameters including septal and lateral TDI-s'as well as global and regional longitudinal strain (LS) at the basal septum were significantly lower in the LVEF $<50 \%$ group than that in the LVEF $\geq 50 \%$ group.

\section{DSE}

DSE results in the patients with $\mathrm{LVEF} \geq 50 \%$ and LVEF $<50 \%$ are shown in Tables 3 and 4 . Forty-three out of $63(68.3 \%)$ in the LVEF $\geq 50 \%$ group and 41 out of 67 (61.2\%) patients in the LVEF $<50 \%$ group were diagnosed as TS-LGAS by DSE. AV Vmax, $\mathrm{PG}_{\text {mean }}$, LVOT $\mathrm{V}_{\max }$, AV velocity ratio, and AVA VTI significantly increased during DSE both in LVEF $\geq 50 \%$ and $<50 \%$ groups (all $\mathrm{P}<0.05$, Tables 3, 4). Systolic blood pressure remained unchanged while peak diastolic blood pressure decreased in both groups during DSE.

In the LVEF $\geq 50 \%$ group, baseline AVA_VTI $\left(0.86 \pm 0.14\right.$ vs. $\left.0.97 \pm 0.14 \mathrm{~cm}^{2}, \mathrm{P}=0.007\right)$ and baseline MAPSE (septal: $7.9 \pm 2.2$ vs. $9.5 \pm 2.2 \mathrm{~mm}, \mathrm{P}=0.009$; 
Table 1 Baseline clinical characteristics

\begin{tabular}{|c|c|c|c|c|}
\hline & $\begin{array}{l}\text { Total } \\
\mathrm{n}=130\end{array}$ & $\begin{array}{l}\mathrm{LVEF} \geq 50 \% \\
\mathrm{n}=63\end{array}$ & $\begin{array}{l}\mathrm{LVEF}<50 \% \\
\mathrm{n}=67\end{array}$ & $\mathrm{P}$ value \\
\hline Age (years) & $78 \pm 8$ & $79 \pm 6$ & $77 \pm 8$ & 0.117 \\
\hline Male [n (\%)] & $83(63.8)$ & $28(44.4)$ & $55(82.1)$ & $<0.001$ \\
\hline BMI $\left(\mathrm{kg} / \mathrm{m}^{2}\right)$ & $27 \pm 4$ & $27 \pm 4$ & $27 \pm 4$ & 0.807 \\
\hline NYHA class [n (\%)] & & & & 0.003 \\
\hline I & $10(7.7)$ & $10(15.9)$ & $0(0)$ & \\
\hline II & $32(24.6)$ & $18(28.6)$ & $14(20.9)$ & \\
\hline III & $75(57.7)$ & $30(47.6)$ & $45(67.2)$ & \\
\hline IV & $13(10.0)$ & $5(7.9)$ & 8 (11.9) & \\
\hline \multicolumn{5}{|l|}{ Comorbidities [n (\%)] } \\
\hline Atrial fibrillation & $59(45.4)$ & $28(44.4)$ & $31(46.3)$ & 0.835 \\
\hline Systemic hypertension & $114(87.7)$ & $55(87.3)$ & $59(88.1)$ & 0.895 \\
\hline Diabetes mellitus & $56(43.1)$ & $21(33.3)$ & $35(52.2)$ & 0.030 \\
\hline Obesity & $28(21.5)$ & $16(25.4)$ & $12(17.9)$ & 0.299 \\
\hline Current smoking & $24(18.5)$ & $9(14.3)$ & $15(22.4)$ & 0.234 \\
\hline Dyslipidemia & $68(52.3)$ & $29(46.0)$ & $39(58.2)$ & 0.165 \\
\hline Coronary heart disease & $79(60.8)$ & $31(49.2)$ & $48(71.6)$ & 0.009 \\
\hline CKD stage III-V & $80(61.5)$ & $38(60.3)$ & $42(62.7)$ & 0.781 \\
\hline Respiratory disease & $35(26.9)$ & $13(20.6)$ & $22(32.8)$ & 0.117 \\
\hline Malignancy & $18(13.8)$ & $9(14.3)$ & $9(13.4)$ & 0.888 \\
\hline Stroke/TIA & $16(12.3)$ & $9(14.3)$ & $7(10.4)$ & 0.506 \\
\hline Creatinine (mg/dl) & $1.15(0.99-1.70)$ & $1.10(0.93-1.53)$ & $1.27(1.00-1.81)$ & 0.040 \\
\hline eGFR (ml/min/1.73qm) & $57(39-74)$ & $57(41-74)$ & $57(36-72)$ & 0.633 \\
\hline C-reactive protein $(\mathrm{mg} / \mathrm{dl})$ & $0.48(0.18-1.04)$ & $0.47(0.14-0.97)$ & $0.53(0.29-1.29)$ & 0.130 \\
\hline \multicolumn{5}{|l|}{ Clinical outcomes } \\
\hline Follow-up duration (months) & 18 (12 to 27$)$ & $20(12$ to 30$)$ & $16(12$ to 27$)$ & 0.226 \\
\hline All-cause death [n (\%)] & $28(21.5)$ & $10(15.9)$ & $18(26.9)$ & 0.128 \\
\hline AVR/TAVI [n (\%)] & $37(28.5) / 44(33.8)$ & $19(30.2) / 22(34.9)$ & $18(26.9) / 22(32.8)$ & 0.813 \\
\hline OP within 30-days [n (\%)] & $35(43.2)$ & $18(46.3)$ & $16(40.0)$ & 0.565 \\
\hline
\end{tabular}

$B M I$ body mass index, NYHA New York Heart Association, $C K D$ chronic kidney disease, eGFR estimated glomerular filtration rate, $A V R$ aortic valve replacement, $T A V I$, transcatheter aortic valve implantation lateral: $9.8 \pm 2.6$ vs. $11.6 \pm 2.2 \mathrm{~mm}, \mathrm{P}=0.011$ ) were significantly lower in the TS-LGAS subgroup than in the PS-LGAS subgroup. During DSE, AV velocity ratio was significantly increased in the PS-LGAS group (baseline $0.25 \pm 0.04$ vs. peak $0.29 \pm 0.05, \mathrm{P}<0.001)$, while remained unchanged in the TS-LGAS group (baseline $0.23 \pm 0.04$ vs. peak $0.24 \pm 0.05, \mathrm{P}=0.531)$. AVA_VTI was significantly increased during DSE in the PS-LGAS group (baseline $0.97 \pm 0.14$ vs. peak $\left.1.23 \pm 0.24 \mathrm{~cm}^{2}, \mathrm{P}<0.001\right)$ and in the TS-LGAS group (baseline $0.86 \pm 0.14$ vs. peak $0.96 \pm 0.20$ $\left.\mathrm{cm}^{2}, \mathrm{P}=0.001\right)$.

In the $\mathrm{LVEF}<50 \%$ group, baseline $\mathrm{AV}$ velocity ratio $(0.19 \pm 0.04$ vs. $0.22 \pm 0.03, P=0.006)$ and baseline AVA_VTI $\left(0.81 \pm 0.18\right.$ vs. $\left.0.94 \pm 0.22 \mathrm{~cm}^{2}, \mathrm{P}=0.008\right)$ were significantly lower, while baseline SPAP $(46 \pm 15$ vs. $37 \pm 14 \mathrm{mmHg}, \mathrm{P}=0.031$ ) was significantly higher in the TS-LGAS group than in the PS-LGAS group. AV velocity ratio (baseline $0.22 \pm 0.03$ vs. peak $0.26 \pm 0.07$,
$\mathrm{P}=0.001$ ) and AVA_VTI (baseline $0.94 \pm 0.22$ vs. peak $\left.1.12 \pm 0.28 \mathrm{~cm}^{2}, \mathrm{P}=0.005\right)$ were significantly increased in the PS-LGAS group, while remained unchanged in the TS-LGAS group during DSE (AV velocity ratio: baseline $0.19 \pm 0.04$ vs. peak $0.19 \pm 0.05, \mathrm{P}=0.352$; AVA_VTI: baseline $0.81 \pm 0.18$ vs. peak $0.80 \pm 0.19 \mathrm{~cm}^{2}, \mathrm{P}=0.742$ ).

All patients with baseline AVAi $\leq 0.3 \mathrm{~cm}^{2} / \mathrm{m}^{2}$ were identified as TS-LGAS by DSE $(n=5)$. In the LVEF $\geq 50 \%$ group, $100 \%, 65.6 \%$ and $57.1 \%$ of patients with a baseline AVAi of $0.31-0.4,0.41-0.5$ and $0.51-0.6 \mathrm{~cm}^{2} / \mathrm{m}^{2}$ were identified as TS-LGAS, respectively. In the LVEF $<50 \%$ group, $78.9 \%, 65.5 \%$, and $25.0 \%$ of patients with a baseline AVAi of $0.31-0.4,0.41-0.5$, and $0.51-0.6 \mathrm{~cm}^{2} /$ $\mathrm{m}^{2}$ were identified as TS-LGAS, respectively. Baseline AVAi $\leq 0.4 \mathrm{~cm}^{2} / \mathrm{m}^{2}$ was highly suggestive of TS-LGAS in LGAS patients with LVEF $\geq 50 \%$ (specificity $100 \%$ and sensitivity $23 \%$ ). 
Table 2 Baseline echocardiographic characteristics

\begin{tabular}{|c|c|c|c|c|}
\hline & $\begin{array}{l}\text { Total } \\
\mathrm{n}=130\end{array}$ & $\begin{array}{l}\mathrm{LVEF} \geq 50 \% \\
\mathrm{n}=63\end{array}$ & $\begin{array}{l}\mathrm{LVEF}<50 \% \\
\mathrm{n}=67\end{array}$ & $P$ value \\
\hline LVEDD (mm) & $50 \pm 7$ & $45 \pm 5$ & $55 \pm 6$ & $<0.001$ \\
\hline IVSd (mm) & $11.2 \pm 1.3$ & $10.7 \pm 1.1$ & $11.7 \pm 1.3$ & $<0.001$ \\
\hline LVPWd (mm) & $10.8 \pm 1.2$ & $10.4 \pm 1.0$ & $11.2 \pm 1.2$ & $<0.001$ \\
\hline LAD (mm) & $44 \pm 6$ & $43 \pm 7$ & $46 \pm 42$ & 0.002 \\
\hline LVEF (\%) & $47 \pm 15$ & $60 \pm 6$ & $46 \pm 4$ & $<0.001$ \\
\hline Septal MAPSE (mm) & $6.8 \pm 2.5$ & $8.2 \pm 2.2$ & $5.4 \pm 1.9$ & $<0.001$ \\
\hline lateral MAPSE (mm) & $8.4 \pm 3.1$ & $10.2 \pm 2.7$ & $6.8 \pm 2.4$ & $<0.001$ \\
\hline TAPSE (mm) & $16.5 \pm 4.9$ & $18.5 \pm 4.6$ & $14.6 \pm 4.4$ & $<0.001$ \\
\hline RVD_basal (mm) & $35 \pm 7$ & $34 \pm 7$ & $37 \pm 7$ & 0.011 \\
\hline RVD_mid (mm) & $31 \pm 8$ & $30 \pm 8$ & $32 \pm 7$ & 0.165 \\
\hline $\mathrm{RAA}\left(\mathrm{cm}^{2}\right)$ & $20 \pm 7$ & $19 \pm 7$ & $21 \pm 7$ & 0.284 \\
\hline $\mathrm{E}(\mathrm{cm} / \mathrm{s})$ & $101 \pm 37$ & $105 \pm 41$ & $97 \pm 31$ & 0.238 \\
\hline DT (ms) & $194 \pm 83$ & $212 \pm 88$ & $176 \pm 74$ & 0.013 \\
\hline $\mathrm{E}^{\prime}(\mathrm{cm} / \mathrm{s})$ & $5.0 \pm 2.2$ & $5.2 \pm 2.1$ & $4.9 \pm 2.2$ & 0.374 \\
\hline $\mathrm{E} / \mathrm{E}^{\prime}$ & $21 \pm 9$ & $21 \pm 10$ & $22 \pm 8$ & 0.354 \\
\hline Diastolic function [n (\%)] & & & & 0.127 \\
\hline Normal & $1(0.8)$ & $1(1.6)$ & 0 & \\
\hline Grade I & $34(26.2)$ & $21(33.3)$ & $13(19.4)$ & \\
\hline Grade II & $59(45.4)$ & $28(44.4)$ & $31(46.3)$ & \\
\hline Grade III & $36(27.7)$ & $13(20.6)$ & $23(34.3)$ & \\
\hline Moderate or severe MR [n (\%)] & $33(25.4)$ & $13(20.6)$ & $20(29.9)$ & 0.228 \\
\hline Moderate or severe AR [n (\%)] & $23(17.7)$ & $11(17.5)$ & $12(17.9)$ & 0.946 \\
\hline Moderate or severe TR [n (\%)] & $37(28.5)$ & $17(27.0)$ & $20(29.9)$ & 0.717 \\
\hline SPAP (mmHg) & $43 \pm 17$ & $43 \pm 18$ & $43 \pm 16$ & 0.944 \\
\hline LVOT (mm) & $22.4 \pm 1.6$ & $21.7 \pm 1.4$ & $23.1 \pm 1.4$ & $<0.001$ \\
\hline $\mathrm{AV} \mathrm{V}_{\max }(\mathrm{m} / \mathrm{s})$ & $3.4 \pm 0.4$ & $3.5 \pm 0.3$ & $3.3 \pm 0.4$ & 0.004 \\
\hline $\mathrm{AV} \mathrm{PG}_{\text {mean }}(\mathrm{mmHg})$ & $29.4 \pm 6.1$ & $31.0 \pm 5.7$ & $27.9 \pm 6.2$ & 0.003 \\
\hline LVOT $\mathrm{V}_{\max }(\mathrm{m} / \mathrm{s})$ & $0.74 \pm 0.14$ & $0.82 \pm 0.12$ & $0.67 \pm 0.12$ & $<0.001$ \\
\hline $\mathrm{AV}$ velocity ratio & $0.22 \pm 0.04$ & $0.24 \pm 0.04$ & $0.20 \pm 0.04$ & $<0.001$ \\
\hline AVA_VTI $\left(\mathrm{cm}^{2}\right)$ & $0.85 \pm 0.15$ & $0.86 \pm 0.14$ & $0.84 \pm 0.16$ & 0.538 \\
\hline Indexed AVA_VTI $\left(\mathrm{cm}^{2} / \mathrm{m}^{2}\right)$ & $0.45 \pm 0.08$ & $0.47 \pm 0.07$ & $0.44 \pm 0.08$ & 0.012 \\
\hline $\mathrm{SVi}\left(\mathrm{ml} / \mathrm{m}^{2}\right)$ & $34.6 \pm 9.2$ & $40.0 \pm 9.2$ & $29.5 \pm 5.6$ & $<0.001$ \\
\hline $\mathrm{Zva}\left(\mathrm{mmHg} / \mathrm{ml} / \mathrm{m}^{2}\right)$ & $4.7 \pm 1.4$ & $4.2 \pm 1.0$ & $5.2 \pm 1.5$ & $<0.001$ \\
\hline Septal TDI-s' $(\mathrm{cm} / \mathrm{s})$ & $3.8 \pm 1.3$ & $4.4 \pm 1.2$ & $3.2 \pm 1.0$ & $<0.001$ \\
\hline lateral TDI-s' (cm/s) & $4.6 \pm 1.5$ & $5.2 \pm 1.4$ & $4.1 \pm 1.5$ & $<0.001$ \\
\hline \multicolumn{5}{|l|}{ Longitudinal strain (\%) } \\
\hline GLS_4ch & $11.4 \pm 4.8$ & $14.9 \pm 3.9$ & $8.2 \pm 2.9$ & $<0.001$ \\
\hline GLS_2ch & $11.6 \pm 4.7$ & $14.7 \pm 3.9$ & $8.6 \pm 3.2$ & $<0.001$ \\
\hline GLS_3ch & $11.5 \pm 4.8$ & $14.7 \pm 4.0$ & $8.7 \pm 3.4$ & $<0.001$ \\
\hline GLS_average & $11.5 \pm 4.5$ & $14.7 \pm 3.6$ & $8.5 \pm 2.8$ & $<0.001$ \\
\hline Basal septal LS & $8.3 \pm 4.0$ & $10.1 \pm 3.6$ & $6.6 \pm 3.6$ & $<0.001$ \\
\hline
\end{tabular}

$L V E D D$ left ventricular end-diastolic dimension, IVSd end-diastolic interventricular septal thickness, $L V P W d$ end-diastolic left ventricular posterior wall thickness, $L V E F$ left ventricular ejection fraction, MAPSE mitral annular plane systolic excursion, TAPSE tricuspid annular plane systolic excursion, $R V D$ right ventricular dimension, $R A A$ end-systolic right atrial area, $E$ mitral inflow early filling velocity, $D T$ deceleration time of early filling, $E^{\prime}$ early diastolic mitral annular velocity, $E / E^{\prime}$ the ratio between mitral inflow early filling velocity and mitral annular velocity, $M R$ mitral regurgitation, $A R$ aortic regurgitation, $T R$ tricuspid regurgitation, SPAP systolic pulmonary artery pressure, LVOT left ventricular outflow tract, $A V A \_V T I$ aortic valve area calculated by the velocity time integral, $A V$ aortic valve, Vmax maximum velocity by continuous-wave Doppler, $P G_{\text {mean }}$ mean transvalvular pressure gradient, $S V i$ stroke volume indexed to body surface area, $Z v a$, valvuloarterial impedance; TDI- $s^{\prime}$ tissue-Doppler imaging derived mitral annular systolic peak velocity, $G L S$ global longitudinal strain 
Table 3 Low-dose dobutamine stress echocardiographic characteristics

\begin{tabular}{|c|c|c|c|c|}
\hline & $\begin{array}{l}\text { Total } \\
n=130\end{array}$ & $\begin{array}{l}\mathrm{LVEF} \geq 50 \% \\
\mathrm{n}=63\end{array}$ & $\begin{array}{l}\text { LVEF }<50 \% \\
n=67\end{array}$ & $P$ value \\
\hline True-severe AS [n (\%)] & $84(64.6)$ & $43(68.3)$ & $41(61.2)$ & 0.400 \\
\hline Pseudo-severe AS [n (\%)] & $46(35.4)$ & $20(31.7)$ & $26(38.8)$ & \\
\hline LV flow reserve $(\Delta \mathrm{SV} \geq 20 \%)[\mathrm{n}(\%)]$ & $76(58.5)$ & $32(50.8)$ & $44(65.7)$ & 0.085 \\
\hline Rest SBP (mmHg) & $126 \pm 21$ & $131 \pm 21$ & $120 \pm 20$ & 0.002 \\
\hline Peak SBP (mmHg) & $125 \pm 28$ & $132 \pm 29$ & $118 \pm 25$ & 0.007 \\
\hline$\Delta \mathrm{SBP}(\%)$ & $0(-14$ to 12$)$ & $-1(-15$ to 13$)$ & $0(-13$ to 8$)$ & 0.658 \\
\hline Rest DBP (mmHg) & $66 \pm 13$ & $66 \pm 13$ & $65 \pm 19$ & 0.820 \\
\hline Peak DBP (mmHg) & $58 \pm 14 *$ & $58 \pm 15^{*}$ & $58 \pm 13^{*}$ & 0.739 \\
\hline$\Delta \mathrm{DBP}(\%)$ & $-11(-23$ to 0$)$ & $-8(-21$ to 0$)$ & $-13(-24$ to 0$)$ & 0.955 \\
\hline Rest HR (beats/min) & $70 \pm 12$ & $67 \pm 11$ & $72 \pm 12$ & 0.006 \\
\hline Peak HR (beats/min) & $92 \pm 20 *$ & $94 \pm 19^{*}$ & $91 \pm 20^{*}$ & 0.385 \\
\hline$\Delta \operatorname{HR}(\%)$ & $29(13$ to 50$)$ & $40(18$ to 61$)$ & 20 (9 to 44$)$ & 0.001 \\
\hline Rest LVEF (\%) & $47 \pm 16$ & $61 \pm 8$ & $34 \pm 8$ & $<0.001$ \\
\hline Peak LVEF (\%) & $58 \pm 17 *$ & $71 \pm 8^{*}$ & $45 \pm 12^{*}$ & $<0.001$ \\
\hline$\Delta$ LVEF $(\%)$ & 24 (12 to 36$)$ & 16 (8 to 25$)$ & $34(20$ to 51$)$ & $<0.001$ \\
\hline Rest LVSV (ml) & $68 \pm 17$ & $74 \pm 16$ & $62 \pm 16$ & $<0.001$ \\
\hline Peak LVSV (ml) & $79 \pm 20 *$ & $85 \pm 20^{*}$ & $73 \pm 19^{*}$ & $<0.001$ \\
\hline$\Delta \operatorname{LVSV}(\%)$ & $23(3$ to 41$)$ & $20(-2$ to 38$)$ & 27 (4 to 42 ) & 0.069 \\
\hline Rest AV V $\max (m / s)$ & $3.4 \pm 0.4$ & $3.5 \pm 0.4$ & $3.3 \pm 0.3$ & 0.001 \\
\hline Peak AV V $\max (m / s)$ & $4.2 \pm 0.6^{*}$ & $4.4 \pm 0.5^{*}$ & $4.0 \pm 0.5^{*}$ & $<0.001$ \\
\hline$\Delta \mathrm{AV} \mathrm{V}_{\max }(\%)$ & 23 (11 to 32$)$ & 27 (15 to 34$)$ & 22 (8 to 32 ) & 0.148 \\
\hline Rest $\mathrm{PG}_{\text {mean }}(\mathrm{mmHg})$ & $30 \pm 6$ & $32 \pm 6$ & $29 \pm 6$ & 0.003 \\
\hline Peak $\mathrm{PG}_{\text {mean }}(\mathrm{mmHg})$ & $45 \pm 12 *$ & $48 \pm 11 *$ & $42 \pm 12^{*}$ & 0.005 \\
\hline$\Delta \mathrm{PG}_{\text {mean }}(\%)$ & 45 (22 to 67$)$ & $48(29$ to 75$)$ & 44 (19 to 66$)$ & 0.685 \\
\hline Rest LVOT V $\mathrm{V}_{\max }(\mathrm{m} / \mathrm{s})$ & $0.7 \pm 0.2$ & $0.8 \pm 0.1$ & $0.7 \pm 0.1$ & $<0.001$ \\
\hline Peak LVOT $V_{\max }(\mathrm{m} / \mathrm{s})$ & $1.0 \pm 0.3^{*}$ & $1.1 \pm 0.2^{*}$ & $0.9 \pm 0.2 *$ & $<0.001$ \\
\hline$\Delta$ LVOT V $_{\max }(\%)$ & $34(9$ to 49$)$ & $36(14$ to 52$)$ & 25 (4 to 43$)$ & 0.063 \\
\hline Rest AV velocity ratio & $0.22 \pm 0.04$ & $0.24 \pm 0.04$ & $0.20 \pm 0.04$ & $<0.001$ \\
\hline Peak AV velocity ratio & $0.24 \pm 0.06^{*}$ & $0.25 \pm 0.06^{*}$ & $0.22 \pm 0.04 *$ & 0.001 \\
\hline$\Delta \mathrm{AV}$ velocity ratio $(\%)$ & $5(-9$ to 19$)$ & $11(-6$ to 18$)$ & $2(-11$ to 21$)$ & 0.479 \\
\hline Rest AVA_VTI $\left(\mathrm{cm}^{2}\right)$ & $0.88 \pm 0.18$ & $0.89 \pm 0.15$ & $0.86 \pm 0.21$ & 0.301 \\
\hline Peak AVA_VTI $\left(\mathrm{cm}^{2}\right)$ & $0.98 \pm 0.27^{*}$ & $1.04 \pm 0.24 *$ & $0.92 \pm 0.27 *$ & 0.009 \\
\hline$\Delta$ AVA_VTI $(\%)$ & $11(-2$ to 28$)$ & 15 (3 to 29$)$ & $4(-9$ to 25$)$ & 0.047 \\
\hline Rest flow rate & $0.22 \pm 0.05$ & $0.24 \pm 0.04$ & $0.21 \pm 0.05$ & 0.003 \\
\hline Peak flow rate & $0.33 \pm 0.10$ & $0.36 \pm 0.10$ & $0.30 \pm 0.08$ & $<0.001$ \\
\hline$\Delta$ flow rate $(\%)$ & 46 (28 to 62$)$ & 49 (33 to 71$)$ & $45(20$ to 60$)$ & 0.151 \\
\hline $\mathrm{AVA}_{\text {proj }}\left(\mathrm{cm}^{2}\right)$ & $0.90 \pm 0.19$ & $0.89 \pm 0.14$ & $0.91 \pm 0.25$ & 0.588 \\
\hline
\end{tabular}

$S B P$ systolic blood pressure, $D B P$ diastolic blood pressure, $H R$ heart rate, $L V E F$ left ventricular ejection fraction, $P G_{\text {mean }}$ mean transvalvular pressure gradient, $S V i$ stroke volume indexed to body surface area, $A V A \_V T I$ aortic valve area calculated by the velocity time integral, LGSAS low-gradient severe aortic stenosis, LGMAS low-gradient moderate aortic stenosis, $A V A_{\text {proj }}$ : projected aortic valve area at normal transvalvular flow rate

$* \mathrm{P}<0.05$ vs. respective parameters at rest
Significant mitral annular calcification (MAC) was found in 80 out of $130(61.5 \%)$ patients. As shown in Fig. 2a, baseline septal MAPSE was significantly lower in the TS-LGAS group as compared to the PS-LGAS group in LVEF $\geq 50 \%$ patients without significant MAC $(8.3 \pm 2.1$ vs. $10.5 \pm 2.0 \mathrm{~mm}, \mathrm{P}=0.005)$. Lateral MAPSE was significantly lower in TS-LGAS group as compared to PS-LGAS group in LVEF $\geq 50 \%$ patients without $(10.7 \pm 2.4$ vs. $12.3 \pm 1.9 \mathrm{~mm}, \mathrm{P}=0.042)$ and with significant $\mathrm{MAC}(8.4 \pm 2.2$ vs. $10.5 \pm 2.3 \mathrm{~mm}, \mathrm{P}=0.048$; Fig. 2b). 
Table 4 DSE characteristics in patients with PS- and TS-LGAS patients

\begin{tabular}{|c|c|c|c|c|}
\hline & \multicolumn{2}{|l|}{$\mathrm{LVEF} \geq 50 \%$} & \multicolumn{2}{|l|}{ LVEF $<50 \%$} \\
\hline & $\begin{array}{l}\text { PS-LGAS } \\
(\mathrm{n}=20)\end{array}$ & $\begin{array}{l}\text { TS-LGAS } \\
(\mathrm{n}=43)\end{array}$ & $\begin{array}{l}\text { PS-LGAS } \\
\mathrm{n}=26\end{array}$ & $\begin{array}{l}\text { TS-LGAS } \\
\mathrm{n}=41\end{array}$ \\
\hline Rest AV V $\max (\mathrm{m} / \mathrm{s})$ & $3.5 \pm 0.4$ & $3.6 \pm 0.4$ & $3.3 \pm 0.4$ & $3.3 \pm 0.3$ \\
\hline Peak AV V $V_{\max }(\mathrm{m} / \mathrm{s})$ & $4.2 \pm 0.5$ & $4.5 \pm 0.5^{*}$ & $3.7 \pm 0.4$ & $4.2 \pm 0.5^{*}$ \\
\hline$\Delta \mathrm{AV} \mathrm{V} \mathrm{V}_{\max }(\%)$ & $18(13$ to 31$)$ & $29(17 \text { to } 36)^{*}$ & 13 (2 to 24$)$ & $26(11 \text { to } 33)^{*}$ \\
\hline Rest $\mathrm{PG}_{\text {mean }}(\mathrm{mmHg})$ & $31 \pm 7$ & $32 \pm 6$ & $28 \pm 6$ & $29 \pm 6$ \\
\hline Peak $\mathrm{PG}_{\text {mean }}(\mathrm{mmHg})$ & $43 \pm 11$ & $50 \pm 11^{*}$ & $35 \pm 8$ & $46 \pm 12^{*}$ \\
\hline$\Delta \mathrm{PG}_{\text {mean }}(\%)$ & 39 (16 to 63$)$ & 54 (36 to 77$)$ & $30(4$ to 49$)$ & $50(28 \text { to } 84)^{*}$ \\
\hline Rest LVOT V $\mathrm{V}_{\max }(\mathrm{m} / \mathrm{s})$ & $0.9 \pm 0.1$ & $0.8 \pm 0.1$ & $0.7 \pm 0.1$ & $0.6 \pm 0.1 *$ \\
\hline Peak LVOT V $V_{\max }(\mathrm{m} / \mathrm{s})$ & $1.2 \pm 0.2$ & $1.1 \pm 0.2 *$ & $1.0 \pm 0.2$ & $0.8 \pm 0.2 *$ \\
\hline$\Delta$ LVOT V $V_{\max }(\%)$ & $38(22$ to 60$)$ & $34(11$ to 50$)$ & $36(10$ to 51$)$ & 23 (3 to 41$)$ \\
\hline Rest AV velocity ratio & $0.25 \pm 0.04$ & $0.23 \pm 0.04$ & $0.22 \pm 0.03$ & $0.19 \pm 0.04^{*}$ \\
\hline Peak AV velocity ratio & $0.29 \pm 0.05$ & $0.24 \pm 0.05^{*}$ & $0.26 \pm 0.07$ & $0.19 \pm 0.04^{*}$ \\
\hline$\Delta \mathrm{AV}$ velocity ratio $(\%)$ & 17 (5 to 26$)$ & $4(-12 \text { to } 16)^{*}$ & $14(-2$ to 36$)$ & $-4(-16 \text { to } 11)^{*}$ \\
\hline Rest AVA_VTI $\left(\mathrm{cm}^{2}\right)$ & $0.97 \pm 0.14$ & $0.86 \pm 0.14 *$ & $0.94 \pm 0.22$ & $0.81 \pm 0.18^{*}$ \\
\hline Peak AVA_VTI $\left(\mathrm{cm}^{2}\right)$ & $1.23 \pm 0.24$ & $0.96 \pm 0.20^{*}$ & $1.12 \pm 0.28$ & $0.80 \pm 0.19 *$ \\
\hline$\Delta$ AVA_VTI $(\%)$ & $20(10$ to 45$)$ & $14(-1 \text { to } 25)^{*}$ & $24(-1$ to 39$)$ & $0(-11 \text { to } 11)^{*}$ \\
\hline Rest septal MAPSE (mm) & $9.5 \pm 2.2$ & $7.9 \pm 2.2^{*}$ & $5.2 \pm 1.6$ & $5.2 \pm 1.6$ \\
\hline Peak septal MAPSE (mm) & $10.2 \pm 2.7$ & $9.2 \pm 2.6$ & $6.2 \pm 2.3$ & $6.2 \pm 2.2$ \\
\hline$\Delta$ septal MAPSE $(\%)$ & $4(-7$ to 18$)$ & $17(0$ to 33$)$ & $17(0$ to 37$)$ & $25(0$ to 50$)$ \\
\hline Rest lateral MAPSE (mm) & $11.6 \pm 2.2$ & $9.8 \pm 2.6^{*}$ & $7.1 \pm 2.3$ & $6.4 \pm 2.4$ \\
\hline Peak lateral MAPSE (mm) & $12.8 \pm 3.4$ & $11.2 \pm 2.7$ & $9.5 \pm 3.2$ & $8.6 \pm 3.3$ \\
\hline$\Delta$ lateral MAPSE $(\%)$ & $12(0$ to 27$)$ & $12(0$ to 28$)$ & 33 (9 to 61$)$ & $37(0$ to 75$)$ \\
\hline Rest TAPSE (mm) & $18.2 \pm 3.9$ & $17.9 \pm 5.0$ & $14.9 \pm 4.9$ & $14.0 \pm 5.4$ \\
\hline Peak TAPSE (mm) & $18.8 \pm 5.6$ & $18.5 \pm 5.3$ & $16.4 \pm 6.0$ & $14.7 \pm 5.5$ \\
\hline$\Delta$ TAPSE $(\%)$ & 5 (- 19 to 23$)$ & $0(-8$ to 17$)$ & $12(-8$ to 20$)$ & $8(-8$ to 18$)$ \\
\hline Rest SPAP (mmHg) & $42 \pm 16$ & $41 \pm 14$ & $37 \pm 14$ & $46 \pm 15^{*}$ \\
\hline Peak SPAP (mmHg) & $55 \pm 16$ & $54 \pm 15$ & $43 \pm 17$ & $57 \pm 15^{*}$ \\
\hline$\Delta \mathrm{SPAP}(\%)$ & $30(11$ to 51$)$ & $34(12$ to 54$)$ & 15 (0 to 29$)$ & $18(10$ to 40$)$ \\
\hline $\operatorname{AVA}_{\text {proj }}\left(\mathrm{cm}^{2}\right)$ & $1.06 \pm 0.04$ & $0.84 \pm 0.11^{*}$ & $1.21 \pm 0.22$ & $0.83 \pm 0.19^{*}$ \\
\hline
\end{tabular}

Abbreviations as shown in Table 3

$* \mathrm{P}<0.05$ vs. PS-LGAS
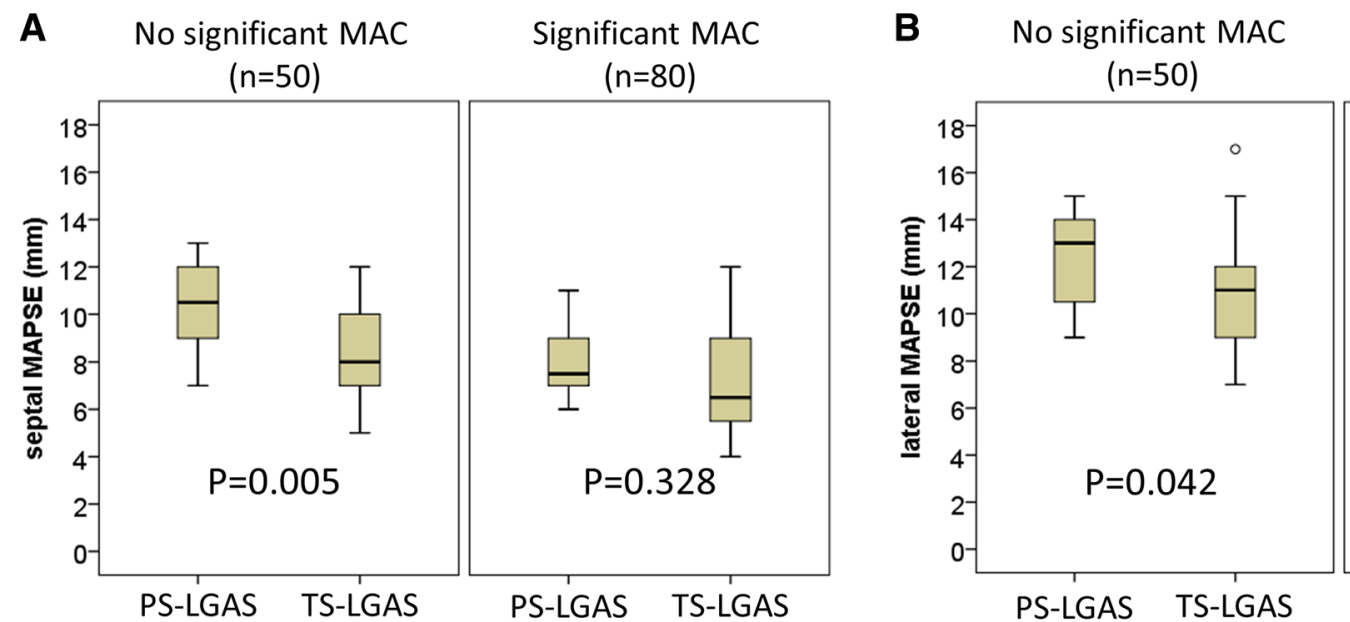

Significant MAC

$(n=80)$

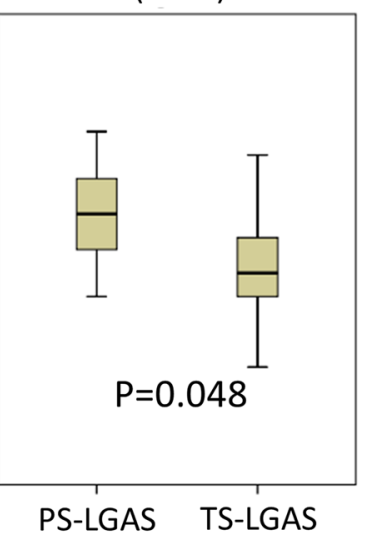

Fig. 2 Septal (a) and lateral (b) mitral annular plane systolic excursion (MAPSE) in low-gradient aortic stenosis (LGAS) patients with and without significant mitral annular calcification (MAC) 


\section{Echocardiographic markers suggestive of TS-LGAS}

Parameters, which are significantly associated with TSLGAS with a statistic difference $(p<0.05)$ between the PS-LGAS and TS-LGAS groups either in the subgroup of LVEF $\geq 50 \%$ or in the subgroup of LVEF $<50 \%$ (Tables 4 , 5 ), were tested in the multivariable binary regression models. Multivariable logical regression models (Table 6) showed that baseline AVA_VTI was an independent determinant of TS-LGAS both in the LVEF $\geq 50 \%$ and $<50 \%$ groups after adjusted for age and sex (LVEF $\geq 50 \%$ : OR 0.45, P=0.004; LVEF < 50\%: OR 0.55, $\mathrm{P}=0.005)$. Besides, lower septal and lateral MAPSE (adjusted OR $0.72-0.75, \mathrm{P}=0.013-0.016)$ as well as septal TDI-s' (adjusted OR 0.53, P =0.010) were significantly associated with TS-LGAS in patients with LVEF $\geq 50 \%$. Higher SPAP and lower AV velocity ratio were associated with TS-LGAS in patients with LVEF < 50\% (SPAP: OR 1.43, $\mathrm{P}=0.045$; AV velocity ratio: $\mathrm{OR} 0.21, \mathrm{P}=0.035)$.

Table 5 Baseline left ventricular longitudinal function characteristics in patients with PS- and TS-LGAS patients

\begin{tabular}{|c|c|c|c|c|}
\hline & \multicolumn{2}{|c|}{$\mathrm{LVEF} \geq 50 \%$} & \multicolumn{2}{|c|}{ LVEF $<50 \%$} \\
\hline & $\begin{array}{l}\text { PS-LGAS } \\
(\mathrm{n}=20)\end{array}$ & $\begin{array}{l}\text { TS-LGAS } \\
(\mathrm{n}=43)\end{array}$ & $\begin{array}{l}\text { PS-LGAS } \\
n=26\end{array}$ & $\begin{array}{l}\text { TS-LGAS } \\
\mathrm{n}=41\end{array}$ \\
\hline $\begin{array}{l}\text { Septal MAPSE } \\
(\mathrm{mm})\end{array}$ & $9.5 \pm 2.2$ & $7.9 \pm 2.2^{*}$ & $5.2 \pm 1.6$ & $5.2 \pm 1.6$ \\
\hline $\begin{array}{l}\text { Lateral MAPSE } \\
\quad(\mathrm{mm})\end{array}$ & $11.6 \pm 2.2$ & $9.8 \pm 2.6^{*}$ & $7.1 \pm 2.3$ & $6.4 \pm 2.4$ \\
\hline $\begin{array}{l}\text { Septal TDI-s' } \\
(\mathrm{cm} / \mathrm{s})\end{array}$ & $5.0 \pm 1.3$ & $4.1 \pm 1.1^{*}$ & $3.3 \pm 1.1$ & $3.0 \pm 0.9$ \\
\hline $\begin{array}{l}\text { Lateral TDI-s' } \\
\quad(\mathrm{cm} / \mathrm{s})\end{array}$ & $5.5 \pm 1.4$ & $5.2 \pm 1.4$ & $4.1 \pm 1.4$ & $4.1 \pm 1.6$ \\
\hline GLS (\%) & $14.4 \pm 3.6$ & $14.9 \pm 3.7$ & $9.0 \pm 2.5$ & $8.3 \pm 3.0$ \\
\hline
\end{tabular}

Abbreviations as shown in Table 2

$* \mathrm{P}<0.05$ vs. PS-LGAS

\section{Clinical Follow up results}

During follow-up, 28 (21.5\%) patients died, 10 (15.9\%) in LVEF $\geq 50 \%$ group [ 3 with TS-LGAS (conservative therapy) and 2, 3, 7 with PS-LGAS (2 with conservative therapy, 3 surgical aortic valve replacement, and 2 transcatheter aortic valve implantation)], and 18 (26.9\%) in LVEF $<50 \%$ group [10 with TS-LGAS (6 conservative therapy, 1, 3, 8 surgical aortic valve replacement, and 3 transcatheter aortic valve implantation) and 8 with PS-LGAS (6 with conservative therapy, 1 surgical aortic valve replacement, and 1 transcatheter aortic valve implantation)].

\section{Discussion}

The major findings of the present study are: (1) DSE appears to also be a helpful tool of defining true-severe low gradient aortic stenosis in patients with LVEF $\geq 50 \%$; (2) Baseline AVA derived from transthoracic echocardiography is an independent determinant of TS-LGAS in both patients with $\mathrm{LVEF} \geq 50 \%$ as well as LVEF $<50 \%$; (3) Reduced septal and lateral MAPSE as well as septal TDI-s' are associated with TS-LGAS in patients with LVEF $\geq 50 \%$ while higher SPAP and lower AV velocity ratio are independently associated with TS-LGAS in patients with LVEF $<50 \%$.

\section{Value of DSE in LGAS patients with reduced and preserved LVEF}

Patients with low gradient severe aortic stenosis (LGAS) and preserved LVEF (AVA $<1 \mathrm{~cm}^{2}, \mathrm{PG}_{\text {mean }}<40 \mathrm{mmHg}$, LVEF $>50 \%$ ) are increasingly recognised in clinical practice. DSE is recommended to define the severity of aortic stenosis in patients with LGAS and reduced LVEF [4, 5]. Our results showed that DES is also useful in defining TSfrom PS-LGAS in patients with LVEF $<50 \%$ (Table 4). This finding is in line with a recent study, which demonstrated that DSE might serve as a valuable tool to distinguish

Table 6 Odds ratio of echocardiographic determinants for low-gradient severe aortic stenosis

\begin{tabular}{|c|c|c|c|c|c|c|}
\hline & \multicolumn{3}{|c|}{$\mathrm{LVEF} \geq 50 \%(\mathrm{n}=63)$} & \multicolumn{3}{|c|}{ LVEF $<50 \%(n=67)$} \\
\hline & $\begin{array}{l}\text { Age and sex } \\
\text { adjusted OR }\end{array}$ & $95 \% \mathrm{CI}$ & $P$ value & $\begin{array}{l}\text { Age and sex } \\
\text { adjusted OR }\end{array}$ & $95 \% \mathrm{CI}$ & $\mathrm{P}$ value \\
\hline Septal MAPSE (per $1 \mathrm{~mm}$ increase) & 0.72 & $0.56-0.93$ & 0.013 & 0.98 & $0.72-1.34$ & 0.906 \\
\hline Lateral MAPSE (per $1 \mathrm{~mm}$ increase) & 0.75 & $0.59-0.95$ & 0.016 & 0.92 & $0.74-1.15$ & 0.473 \\
\hline Septal TDI-s' (per $1 \mathrm{~cm} / \mathrm{s}$ increase) & 0.53 & $0.33-0.86$ & 0.010 & 0.73 & $0.44-1.21$ & 0.219 \\
\hline SPAP (per $10 \mathrm{mmHg}$ increase) & 0.95 & $0.70-1.28$ & 0.732 & 1.43 & $1.01-2.04$ & 0.045 \\
\hline AVA_VTI (per $0.1 \mathrm{~cm}^{2}$ increase) & 0.45 & $0.26-0.78$ & 0.004 & 0.55 & $0.36-0.83$ & 0.005 \\
\hline AV velocity ratio (per 0.1 increase) & 0.29 & $0.06-1.29$ & 0.286 & 0.21 & $0.05-0.90$ & 0.035 \\
\hline
\end{tabular}

CI confidence interval, abbreviations as shown in Table 2 
TS-LGAS from PS-LGAS in patients with paradoxical LGAS [14].

There were 20 out of 63 (31.7\%) LGAS patients with preserved LVEF presenting with paradoxical low-flow LGAS $\left(\mathrm{SVi} \leq 35 \mathrm{ml} / \mathrm{m}^{2}\right)$ in our cohort. We compared other related parameters as well as the response on DSE between the two subgroups (low-flow vs. normal-flow LGAS). The data show that LV cavity in these patients was smaller than in patients with normal-flow LGAS (left ventricular end-diastolic dimension $43 \pm 4$ vs. $46 \pm 5 \mathrm{~mm}, \mathrm{P}=0.021$ ). Moreover, $\mathrm{DSE}$ seems also to be feasible to identify the TS-AS patients in the low-flow LGAS subgroup as in the normal-flow LGAS subgroup (Supplementary Table 1). Future studies with larger patient cohort are warrant to verify these results.

\section{Echocardiographic determinant of TS-LGAS in patients with preserved LVEF}

In line with previous findings from our group [15] and others [16], the present study results demonstrate that reduced MAPSE is suggestive of TS-LGAS in patients with LVEF $>50 \%$. A previous study also showed that MAPSE correlated with aortic valve area (Spearman $r=0.18, \mathrm{P}=0.02$ ) in 205 asymptomatic AS patients with LVEF $\geq 50 \%$ [17]. In another study, Rydberg and colleagues found that left atrioventricular plane displacement (MAPSE), but not left ventricular ejection fraction, was influenced by the degree of aortic stenosis [16]. In patients with LGAS and preserved LVEF, LVEF is maintained at normal range at the expense of high LV end-diastolic pressure and volume. In this circumference, LV cannot recruit additional preload because the maximum sarcomere length is already reached and the hypertrophied stiff ventricle could not tolerate additional filling load [18]. This might explain why reduced MAPSE, as a function of LV hemodynamic load, was revealed as the most sensitive marker of TS-LGAS in LGAS patients with LVEF $>50 \%$. Additionally, reduction in TDI-s', another parameter reflecting LV longitudinal dysfunction, is also suggestive of TS-LGAS in AS patients with $\mathrm{LVEF} \geq 50 \%$.

\section{Echocardiographic determinants of TS-LGAS in patients with reduced LVEF}

In LGAS patients with reduced LVEF, the disease features include both AS and heart failure. Ventricular remodeling (myocyte hypertrophy and myocardial fibrosis), systolic and diastolic dysfunction, in the setting of valvular stenosis and heart failure scenarios, contributes to the progressively elevated LV filling pressure, and/or left atrial pressure, which in turn would transmit to the pulmonary vasculature, inducing pulmonary venous congestion and pulmonary hypertension, so called WHO Group II - pulmonary hypertension [19, 20].
A previous study showed that pulmonary hypertension was presented in the majority of patients with severe aortic stenosis, and ejection fraction serves as one of the correlates of pulmonary hypertension in patients with severe aortic stenosis [21]. Another study indicated that both smaller aortic valve area and lower LVEF were responsible for the presence of pulmonary hypertension in a cohort of 626 patients with severe aortic stenosis [22]. In line with above findings, we found that increased systolic pulmonary pressure is an independent determinant of TS in LGAS patients with reduced LVEF. Another finding of the present study is that lower $\mathrm{AV}$ velocity ratio is related to the presence of true severe in LGAS patients with reduced LVEF. This parameter is only recommended in the EAE/ASE recommendations, a value of $<0.25$ is suggestive of severe AS [23]. In our cohort, this value ranged from 0.23 at rest and 0.24 during DSE in TS-LGAS patients with LVEF $>50 \%$, and 0.19 at rest and 0.19 during DSE in TS-LGAS patients with reduced LVEF. A previous study reported that velocity ratios could not only define the severity of AS, but also predict outcomes in patients with TS-LGAS and preserved LVEF [24]. Our results suggest AV velocity ratio could be used as an independent predictor for defining TS in LGAS patients with reduced LVEF.

\section{Limitations}

This retrospective study was performed monocentric and the number of patients included in the sub-cohorts was relatively small. The results of the present study might thus be affected by patient selection bias. Future studies with large patient cohort are warranted to validate the results obtained from this study. In this study, projected aortic valve area assessed by DSE was used to define the severity of AS [25]. Other imaging modalities may aid in the diagnosis of severe AS. Macroscopic evaluation of the valve at the time of valve replacement or measurement of aortic valve calcium score by multislice computed tomography could provide additional accuracy to differ TS- from PS-AS [26]. Due to the lack of available CT data for AV calcium score in our cohort, the severity of AS was only determined by projected aortic valve area. This study limitation should be considered in the interpretation of results derived from the current study.

\section{Clinical implications}

Our study results suggest that DSE enables evaluation of the severity of AS both in LGAS patients with reduced LVEF and in those with preserved LVEF, which is consistent with what has been demonstrated by previous studies $[13,26]$. In the present study, we further explored the echocardiographic determinants suggestive of TS-LGAS. Reduced MAPSE and 
TDI-s' are suggestive of TS in LGAS patients with preserved LVEF, while increased SPAP and lower AV velocity ratio are suggestive of TS in LGAS patients with reduced LVEF. These findings provide incremental information on the diagnosis and therapy decision for LGAS patients, particularly in those patients who cannot tolerate DSE due to the presence of contraindications. Patients with related echocardiographic features should be carefully evaluated for the AS severity with alternative complementary imaging modalities, such as measurement of aortic valve calcium score assessed by multislice computed tomography.

\section{Conclusions}

DSE is valuable for staging of LGAS in patients with both reduced and preserved LVEF. Low longitudinal LV function (MAPSE) and septal TDI-s' are associated with TS-LGAS in patients with preserved LVEF, and high trans-tricuspid pressure gradient (SPAP) and low AV velocity ratio are associated with TS-LGAS in patients with reduced LVEF.

Acknowledgements This work was supported by grants from the Bundesministerium für Bildung und Forschung of the Federal Republic of Germany (BMBF 01EO1504 MO.6).

\section{Compliance with ethical standards}

Conflict of interest All authors have no conflict of interest.

Ethical approval The study was conducted in accordance to the Declaration of Helsinki and approved by the Local Ethics Committee at the University of Würzburg (AZ 11/03 and 60/14). Informed consent was obtained from all patients or their guardians.

Open Access This article is distributed under the terms of the Creative Commons Attribution 4.0 International License (http://creativeco mmons.org/licenses/by/4.0/), which permits unrestricted use, distribution, and reproduction in any medium, provided you give appropriate credit to the original author(s) and the source, provide a link to the Creative Commons license, and indicate if changes were made.

\section{References}

1. Bonow RO, Carabello BA, Kanu C, de Leon AC Jr, Faxon DP, Freed MD, Gaasch WH, Lytle BW, Nishimura RA, O'Gara PT, O'Rourke RA, Otto CM, Shah PM, Shanewise JS, Smith SC Jr, Jacobs AK, Adams CD, Anderson JL, Antman EM, Fuster V, Halperin JL, Hiratzka LF, Hunt SA, Nishimura R, Page RL, Riegel B (2006) ACC/AHA 2006 guidelines for the management of patients with valvular heart disease: a report of the American College of Cardiology/American Heart Association Task Force on Practice Guidelines (writing committee to revise the 1998 Guidelines for the Management of Patients With Valvular Heart Disease): developed in collaboration with the Society of Cardiovascular Anesthesiologists: endorsed by the Society for Cardiovascular
Angiography and Interventions and the Society of Thoracic Surgeons. Circulation 114:e84-e231. https://doi.org/10.1161/CIRCU LATIONAHA.106.176857

2. Clavel MA, Dumesnil JG, Capoulade R, Mathieu P, Senechal M, Pibarot P (2012) Outcome of patients with aortic stenosis, small valve area, and low-flow, low-gradient despite preserved left ventricular ejection fraction. J Am Coll Cardiol 60:1259-1267. https ://doi.org/10.1016/j.jacc.2011.12.054

3. Hachicha Z, Dumesnil JG, Bogaty P, Pibarot P (2007) Paradoxical low-flow, low-gradient severe aortic stenosis despite preserved ejection fraction is associated with higher afterload and reduced survival. Circulation 115:2856-2864. https://doi. org/10.1161/CIRCULATIONAHA.106.668681

4. Grayburn PA (2006) Assessment of low-gradient aortic stenosis with dobutamine. Circulation 113:604-606. https://doi. org/10.1161/CIRCULATIONAHA.105.600684

5. Nishimura RA, Otto CM, Bonow RO, Carabello BA, Erwin JP, Guyton RA, O'Gara PT, Ruiz CE, Skubas NJ, Sorajja P, Sundt TM, Thomas JD, Anderson JL, Halperin JL, Albert NM, Bozkurt B, Brindis RG, Creager MA, Curtis LH, DeMets D, Hochman JS, Kovacs RJ, Ohman EM, Pressler SJ, Sellke FW, Shen WK, Stevenson WG, Yancy CW (2014) 2014 AHA/ACC guideline for the management of patients with valvular heart disease: a report of the American College of Cardiology/American Heart Association Task Force on Practice Guidelines. J Thorac Cardiovasc Surg 148:e1-e132. https://doi.org/10.1016/j. jtcvs.2014.05.014

6. Cheitlin MD, Armstrong WF, Aurigemma GP, Beller GA, Bierman FZ, Davis JL, Douglas PS, Faxon DP, Gillam LD, Kimball TR, Kussmaul WG, Pearlman AS, Philbrick JT, Rakowski H, Thys DM, Antman EM, Smith SC Jr, Alpert JS, Gregoratos G, Anderson JL, Hiratzka LF, Hunt SA, Fuster V, Jacobs AK, Gibbons RJ, Russell RO (2003) ACC/AHA/ASE 2003 guideline update for the clinical application of echocardiography: summary article: a report of the American College of Cardiology/American Heart Association Task Force on Practice Guidelines (ACC/AHA/ASE Committee to Update the 1997 Guidelines for the Clinical Application of Echocardiography). Circulation 108:1146-1162. https ://doi.org/10.1161/01.CIR.0000073597.57414.A9

7. Lang RM, Badano LP, Mor-Avi V, Afilalo J, Armstrong A, Ernande L, Flachskampf FA, Foster E, Goldstein SA, Kuznetsova T, Lancellotti P, Muraru D, Picard MH, Rietzschel ER, Rudski L, Spencer KT, Tsang W, Voigt JU (2015) Recommendations for cardiac chamber quantification by echocardiography in adults: an update from the American Society of Echocardiography and the European Association of Cardiovascular Imaging. J Am Soc Echocardiogr 28:1-39 e14. https://doi.org/10.1016/j. echo.2014.10.003

8. Nagueh SF, Appleton CP, Gillebert TC, Marino PN, Oh JK, Smiseth OA, Waggoner AD, Flachskampf FA, Pellikka PA, Evangelista A (2009) Recommendations for the evaluation of left ventricular diastolic function by echocardiography. $\mathrm{J}$ Am Soc Echocardiogr 22:107-133. https://doi.org/10.1016/j. echo.2008.11.023

9. Chahal NS, Lim TK, Jain P, Chambers JC, Kooner JS, Senior R (2010) Normative reference values for the tissue Doppler imaging parameters of left ventricular function: a population-based study. Eur J Echocardiogr 11:51-56. https://doi.org/10.1093/ejechocard /jep164

10. Liu D, Hu K, Stork S, Herrmann S, Kramer B, Cikes M, Gaudron PD, Knop S, Ertl G, Bijnens B, Weidemann F (2014) Predictive value of assessing diastolic strain rate on survival in cardiac amyloidosis patients with preserved ejection fraction. PloS ONE 9:e115910. https://doi.org/10.1371/journal.pone.0115910

11. Baumgartner H, Hung J, Bermejo J, Chambers JB, Evangelista A, Griffin BP, Iung B, Otto CM, Pellikka PA, Quinones M (2009) 
Echocardiographic assessment of valve stenosis: EAE/ASE recommendations for clinical practice. Eur J Echocardiogr 10:1-25. https://doi.org/10.1093/ejechocard/jen303

12. Nishimura RA, Grantham JA, Connolly HM, Schaff HV, Higano ST, Holmes DR Jr (2002) Low-output, low-gradient aortic stenosis in patients with depressed left ventricular systolic function: the clinical utility of the dobutamine challenge in the catheterization laboratory. Circulation 106:809-813

13. Clavel MA, Burwash IG, Mundigler G, Dumesnil JG, Baumgartner H, Bergler-Klein J, Senechal M, Mathieu P, Couture C, Beanlands R, Pibarot P (2010) Validation of conventional and simplified methods to calculate projected valve area at normal flow rate in patients with low flow, low gradient aortic stenosis: the multicenter TOPAS (True or Pseudo Severe Aortic Stenosis) study. J Am Soc Echocardiogr 23:380-386. https://doi. org/10.1016/j.echo.2010.02.002

14. Clavel MA, Ennezat PV, Marechaux S, Dumesnil JG, Capoulade R, Hachicha Z, Mathieu P, Bellouin A, Bergeron S, Meimoun P, Arsenault M, Le Tourneau T, Pasquet A, Couture C, Pibarot P (2013) Stress echocardiography to assess stenosis severity and predict outcome in patients With paradoxical low-flow, low-gradient aortic stenosis and preserved LVEF. Jacc Cardiovasc Imag 6:175-183. https://doi.org/10.1016/j.jcmg.2012.10.015

15. Herrmann S, Stork S, Niemann M, Lange V, Strotmann JM, Frantz S, Beer M, Gattenlohner S, Voelker W, Ertl G, Weidemann F (2011) Low-gradient aortic valve stenosis myocardial fibrosis and its influence on function and outcome. J Am Coll Cardiol 58:402-412. https://doi.org/10.1016/j.jacc.2011.02.059

16. Rydberg E, Gudmundsson P, Kennedy L, Erhardt L, Willenheimer R (2004) Left atrioventricular plane displacement but not left ventricular ejection fraction is influenced by the degree of aortic stenosis. Heart 90:1151-1155. https://doi.org/10.1136/ hrt.2003.020628

17. Matos JD, Kiss JE, Locke AH, Goldberg RI, Mukamal K, Manning WJ (2017) Relation of the mitral annular plane systolic excursion to risk for intervention in initially asymptomatic patients with aortic stenosis and preserved systolic function. Am J Cardiol 120:2031-2034. https://doi.org/10.1016/j.amjcard.2017.08.021

18. Ross J Jr (1985) Afterload mismatch in aortic and mitral valve disease: implications for surgical therapy. J Am Coll Cardiol 5:811-826

19. Fang JC, DeMarco T, Givertz MM, Borlaug BA, Lewis GD, Rame JE, Gomberg-Maitland M, Murali S, Frantz RP, McGlothlin D, Horn EM, Benza RL (2012) World Health Organization pulmonary hypertension group 2: pulmonary hypertension due to left heart disease in the adult-a summary statement from the pulmonary hypertension Council of the International Society for heart and lung transplantation. J Heart Lung Transplant 31:913-933. https://doi.org/10.1016/j.healun.2012.06.002

20. Pellegrini P, Rossi A, Pasotti M, Raineri C, Cicoira M, Bonapace S, Dini FL, Temporelli PL, Vassanelli C, Vanderpool R, Naeije R, Ghio S (2014) Prognostic relevance of pulmonary arterial compliance in patients with chronic heart failure. Chest 145:1064-1070. https://doi.org/10.1378/chest.13-1510

21. Ben-Dor I, Goldstein SA, Pichard AD, Satler LF, Maluenda G, Li Y, Syed AI, Gonzalez MA, Gaglia MA Jr, Wakabayashi K, Delhaye C, Belle L, Wang Z, Collins SD, Torguson R, Okubagzi P, Aderotoye A, Xue Z, Suddath WO, Kent KM, Epstein SE, Lindsay J, Waksman R (2011) Clinical profile, prognostic implication, and response to treatment of pulmonary hypertension in patients with severe aortic stenosis. Am J Cardiol 107:1046-1051. https://doi. org/10.1016/j.amjcard.2010.11.031

22. Kapoor N, Varadarajan P, Pai RG (2008) Echocardiographic predictors of pulmonary hypertension in patients with severe aortic stenosis. Eur J Echocardiogr 9:31-33. https://doi.org/10.1016/j. euje.2007.01.005

23. Baumgartner H, Hung J, Bermejo J, Chambers JB, Evangelista A, Griffin BP, Iung B, Otto CM, Pellikka PA, Quinones M (2009) Echocardiographic assessment of valve stenosis: EAE/ASE recommendations for clinical practice. J Am Soc Echocardiogr 22:123. https://doi.org/10.1016/j.echo.2008.11.029 quiz 101-102.

24. Jander N, Hochholzer W, Kaufmann BA, Bahlmann E, Gerdts E, Boman K, Chambers JB, Nienaber CA, Ray S, Rossebo A, Pedersen TR, Wachtell K, Gohlke-Barwolf C, Neumann FJ, Minners J (2014) Velocity ratio predicts outcomes in patients with low gradient severe aortic stenosis and preserved EF. Heart 100:1946-1953. https://doi.org/10.1136/heartjnl-2014-305763

25. Annabi MS, Touboul E, Dahou A, Burwash IG, Bergler-Klein J, Enriquez-Sarano M, Orwat S, Baumgartner H, Mascherbauer J, Mundigler G, Cavalcante JL, Larose E, Pibarot P, Clavel MA (2018) Dobutamine stress echocardiography for management of low-flow, low-gradient aortic stenosis. J Am Coll Cardiol 71:475485. https://doi.org/10.1016/j.jacc.2017.11.052

26. Clavel MA, Ennezat PV, Marechaux S, Dumesnil JG, Capoulade R, Hachicha Z, Mathieu P, Bellouin A, Bergeron S, Meimoun P, Arsenault M, Le Tourneau T, Pasquet A, Couture C, Pibarot P (2013) Stress echocardiography to assess stenosis severity and predict outcome in patients with paradoxical low-flow, lowgradient aortic stenosis and preserved LVEF. JACC Cardiovasc Imaging 6:175-183. https://doi.org/10.1016/j.jcmg.2012.10.015 